\title{
ENTRENAMIENTO DEL VIBRATO EN CANTANTES
}

\author{
Vibrato training in singers
}

Marco Antonio Guzmán ${ }^{(1)}$

\begin{abstract}
RESUMEN
Tema: el vibrato de la voz es uno de los rasgos acústicos, técnicos y estilísticos más importantes en la caracterización de la cualidad de la voz en cantantes clásicos. Técnicamente, vibrato es una pulsación del tono, usualmente acompañada con pulsaciones sincrónicas de intensidad y timbre. El vibrato puede ser descrito acústicamente por el rate, extent y periodicidad de las modulaciones en frecuencia y modulaciones de la amplitud de la voz. El objetivo del presente artículo es mostrar y explicar un nuevo procedimiento para entrenar y corregir vibrato en cantantes. Procedimientos: estudiante de canto lirico de 25 años, sexo masculino con clasificación vocal de barítono lirico, voz normal desde el punto de vista perceptual. La corrección del vibrato se realiza por medio del método "Entrenamiento rítmico del vibrato". Se produce la subdivisión rítmica de un pulso durante la emisión de una vocal con un "tempo" o velocidad constante. Posteriormente se varía la velocidad del pulso, las vocales y la melodía del ejercicio. Los parámetros acústicos del vibrato son evaluados objetivamente antes y después del entrenamiento. Resultados: el paciente logró producir un vibrato con rate, extent y regularidad dentro de rangos normales. Esto se realizó de forma consciente y dirigida, utilizando actividad laríngea y abdominal en forma conjunta. La naturalidad del vibrato continuó mejorando después de finalizado el entrenamiento. Conclusiones: es posible el entrenamiento y/o corrección del vibrato en forma dirigida a través de ejercicios específicos destinados a ese objetivo. El apoyo visual cumple un rol importante en los resultados obtenidos.
\end{abstract}

DESCRIPTORES: Voz; Calidad de la Voz; Entrenamiento de la Voz

\section{INTRODUCCIÓN}

El vibrato de la voz es considerado uno de los rasgos más característicos y esenciales del canto clásico. Seashore ${ }^{1}$ fue el primero en estudiar este fenómeno vocal en forma extensa y en proveer una definición de vibrato. Según este autor: "vibrato es una pulsación del tono, usualmente acompañada con pulsaciones sincrónicas de intensidad y timbre de tal forma que el extent y el rate den una agradable flexibilidad y riqueza a los tonos".

El vibrato puede ser descrito acústicamente por el rate, extent y periodicidad de las modulaciones en frecuencia y modulaciones de la amplitud de la voz. El rate se describe como cuan rápida son las modulaciones y es expresado en $\mathrm{Hz}$. Típicamente los valores van desde 4 a $7 \mathrm{~Hz}$. Hirano ${ }^{2}$ midió el rate en 23 cantantes y encontró que éste varía entre 4,1

(1) Académico Escuela de Fonoaudiología de la Facultad de Medicina de la Universidad de Chile

Conflito de interesses: inexistente y 6,6 Hz. En otro estudio más reciente, se encontró que el promedio de 22 cantantes fue $5,5 \mathrm{~Hz}^{3}$.

El extent describe cuan largo o corto son las modulaciones relativas al promedio de la frecuencia o amplitud durante el ciclo del vibrato. El promedio de extent en un vibrato técnicamente bien realizado debería ser de un semitono (100 cents) ${ }^{4}$. Sin embargo, este parámetro varía mucho dependiendo del cantante y de la expresión vocal ${ }^{5}$. Howes ${ }^{6}$ encontró que el promedio del extent en cantantes profesionales varía entre 0,41 y 1,58 semitonos.

El vibrato siempre ha sido asociado con la calidad y la belleza de la voz del cantante, es así como Robinson ${ }^{7}$ concluye que las voces más bellas tienen rasgos acústicos en común incluyendo un rate de vibrato constante y suave, con un extent moderado.

La regularidad del vibrato señala la estabilidad o repetitividad de las modulaciones. La regularidad es considerada el mejor reflejo de la habilidad de un cantante. Mientras más regular es el vibrato, la voz es percibida más bella y de mejor calidad ${ }^{8}$. 
Esto significa que el vibrato es mejor a medida que tenga el rate y el extent más constantes y regulares en el tiempo ${ }^{4,9}$. En un estudio reciente, realizado con voces de tenores famosos, el vibrato jitter fue el parámetro que más afectó la valoración perceptual del mismo, existiendo una correlación negativa entre jitter y calidad del vibrato, es decir, a menor valor jitter de vibrato (mayor regularidad), la calidad de éste es mayor ${ }^{10}$.

Otro rasgo acústico del vibrato es el "onset", el cual es considerado como el tiempo en segundos que tarda el inicio de la primera oscilación del vibrato desde que partió la emisión de sonido. Estudios asocian el retardo del inicio vibrato (mayor a 0,5 segundos) con una percepción de un vibrato pobre y esto también es altamente correlacionado con una pobre calidad de la voz en el canto ${ }^{11,12}$. Otros autores indican que mientras más temprano es el vibrato onset, más amplio es el extent ${ }^{6}$.

Acústicamente, el vibrato involucra modulaciones de la frecuencia y modulaciones de amplitud. En relación a esto, Horiii ${ }^{13}$ plantea que en la modulación de F0, los armónicos también cambian proporcionalmente. Esto causa que armónicos individuales se acerquen o alejen alternadamente de los picos de las resonancias del tracto vocal, resultando un incremento o disminución de la presión de sonido (SPL) de la voz al salir de la boca. Similarmente Schutte ${ }^{14}$ estudió las propiedades espectrales acústicas de las notas agudas de un tenor y encontró que el timbre y la amplitud del vibrato, planteados desde un comienzo por Seashore, pueden resultar de los movimientos periódicos de los armónicos de la fuente de voz a través de los formantes del tracto vocal. Imaizumi ${ }^{15}$, demostró que además de este mecanismo, las fluctuaciones del espectro de la fuente de voz y las vibraciones de las paredes del tracto vocal pueden contribuir con las características acústicas del vibrato. Al parecer el mecanismo primario del vibrato del canto clásico es la modulación de F0, y las modulaciones en amplitud y timbre surgen desde la interacción acústica entre los armónicos y las resonancias del tracto vocal. Esto parece ser soportado por los datos de Horii ${ }^{16}$, quien encontró que la modulación de frecuencia durante el vibrato fue mucho más regular que la modulación de amplitud.

Desde el punto de vista fisiológico, el vibrato ha sido generalmente atribuido a fluctuaciones en la tensión de las cuerdas vocales, siendo esto un resultado de la modulación de la actividad del músculo cricotiroideo (CT) ${ }^{15,17}$.

Hirano ${ }^{18}$ encontró contracciones rítmicas en varios músculos laríngeos coincidentes con las oscilaciones del vibrato. El músculo CT fue el que presentó mayor participación. Existe también participación en menor grado del tiroaritenoideo (TA) y el crocoaritenoideo lateral (CAL).

Por otro lado, Appelman ${ }^{19}$ observó la actividad diafragmática y abdominal utilizando fluoroscopía y EMG de superficie. Los sujetos estudiados mostraron movimientos abdominales ascendentes en fase con el aumento de la F0 durante los ciclos del vibrato. Paralelamente, la EMG de los músculos abdominales mostró actividad coincidente con el vibrato rate. Los autores concluyeron que el vibrato fue controlado principalmente por los músculos abdominales más que por la actividad muscular laríngea en este caso.

El vibrato puede ser separado en dos tipos de producción. La primera se relaciona con la producción rítmica de pulsaciones de presión subglótica producida por contracciones de los músculos abdominales, para modular la frecuencia fundamental. Parece ser que para este tipo de vibrato la contracción de los músculos laríngeos se mantiene estable en contra de la actividad de los músculos abdominales. El segundo tipo de vibrato, y probablemente el más común, es el mediado por la laringe, en el cual la modulación de F0 resulta principalmente de la modulación de la actividad del CT. Estas oscilaciones musculares se manifiestan por si solas cuando los aspectos estructurales de las cuerdas vocales en coordinación con el mecanismo respiratorio, son balanceados apropiadamente y el sistema neuromuscular es suficientemente maduro para producir contracciones regulares de músculos seleccionados.

Ambos tipos de vibrato ofrecen diferentes opciones a los cantantes, por ejemplo el método abdominal permite que los cantantes puedan controlar el rate del vibrato de mejor forma que utilizando sólo la musculatura laríngea ${ }^{20}$. Laukkanen ${ }^{21}$ sugiere que los cantantes producen vibrato utilizando uno de los dos tipos de producción o la combinación de ambos en distinto grado.

Existe un acuerdo general en la comunidad del canto que el vibrato es un rasgo de la voz de los cantantes entrenados que ocurre naturalmente cuando la voz es producida con libertad y buena técnica ${ }^{6}$. Los cantantes profesionales parecen desarrollar el vibrato automáticamente, sin esforzarse en forma activa por adquirirlo, de hecho, rara vez los profesores de canto dan instrucciones a sus alumnos acerca de su vibrato a menos que exista un problema. En un estudio longuitudinal, se examinó el vibrato de 22 estudiantes de canto al comienzo y después de 3 años de estudio profesional. Los resultados mostraron que las voces con vibrato lento después de tres años aumentaron su rate, mientras que las voces con vibrato rápido, disminuyeron el rate, asi mismo, las voces con vibrato 
irregular mostraron mayor regularidad al final de la educación ${ }^{3}$. Esto implica que el vibrato se regula y se desarrolla con el avance de la técnica vocal.

Existen profesores que consideran inapropiado intentar cambiar voluntariamente los rasgos del vibrato. Hay datos experimentales que muestran la inhabilidad de cantantes profesionales para cambiar su vibrato rate, incluso cuando se les pide hacerlo ${ }^{22}$. Por otra parte, otros profesores si creen posible y adecuado desarrollar o corregir un vibrato inapropiado en sus alumnos, a través de ejercicios específicos ${ }^{23}$. Respaldando esto, otros estudios han encontrado que los cantantes si son capaces de cambiar el vibrato rate en forma deliberada ${ }^{24}$.

El objetivo del presente artículo es mostrar y explicar en forma detallada un nuevo procedimiento para entrenar y corregir vibrato en cantantes. Se presenta el caso de un estudiante de canto que a pesar de llevar 5 años estudiando, no había logrado desarrollar un vibrato técnicamente correcto. El procedimiento propuesto por el autor, involucra los dos aspectos fisiológicos del vibrato ya descritos: acción de la musculatura laríngea y participación de la musculatura abdominal.

\section{PRESENTACION DEL CASO}

Estudiante de canto lirico de 25 años, sexo masculino con clasificación vocal de barítono lirico. El sujeto en estudio se encuentra cursando el 5 año de canto lirico. Su desarrollo vocal en voz cantada ha seguido un curso normal en todos sus parámetros, excepto en el desarrollo del vibrato. El profesor de canto le indica que no intente crear un vibrato artificial, sino que espere un desarrollo natural de éste con el paso del tiempo paralelamente la adquisición gradual de la técnica vocal.

Los resultados de la videoestroboscopía laríngea muestran una laringe anatómicamente normal, solo se observa leve irritación en la zona aritenoidea probablemente asociada a reflujo faringolaríngeo. En la valoración laringoscópica con fibra flexible se observa leve constricción aritenoepiglotica al emitir notas en la zona aguda de la tesitura en registro de cabeza (voz cubierta o full head).

La evaluación vocal hablada no muestra anormalidades. En la valoración vocal de voz cantada, perceptualmente el paciente presenta un leve grado de voz con esfuerzo en la emisión en extremo superior de la tesitura (últimos dos semitonos), hecho que confirma lo observado en la laringoscopía flexible. La tesitura del sujeto se encuentra en el rango de Sol 1 - La bemol 3. El pasaje vocal de registro de pecho a cabeza (voz cubierta) está alrededor de $\operatorname{Re} \# 3$, no presentando dificultades técnicas. Esta altura varía según la vocal utilizada, obteniéndose el valor más alto en la vocal abierta /a:/ (Mi 3) y el valor más bajo para la vocal cerrada /u:/ (Re 3). Los parámetros respiratorios no presentan alteraciones durante la emisión de voz cantada. Se destaca el buen manejo del apoyo respiratorio y "fiato" que el paciente presenta durante la producción diferentes vocalizaciones de dificultad técnica variable. Subjetivamente su voz presenta una gran sonoridad y brillo (voz resonante), siendo estos aspectos excesivos en la mayoría de la ocasiones. Según información entregada por el sujeto uno de sus problemas técnicos e interpretativos es el manejo de la dinámica (variación de la intensidad con fines expresivos), señalando que su principal dificultad en este ámbito es la emisión de emisiones de baja intensidad.

Técnicamente, el vibrato se percibe con anormalidades (considerando el tipo repertorio que el paciente borda), siendo las más importantes desde el punto de vista perceptual, la irregularidad y la velocidad aumentada de las oscilaciones ("vibrato caprino"). Los parámetros acústicos del vibrato fueron valorados objetivamente mediante el programa VoceVista.

Emision vocal /a/ sostenida: Rate $=7,5 \mathrm{~Hz}$, Extent $=80$ cents, Onset $=52 \mathrm{~ms}$ y vibrato jitter $=$ $8,6 \%$ (Figuras 1 y 2). Emisión de vocal /a/ de la palabra "amarilli" (canción amarilli), Rate $=7,8 \mathrm{~Hz}$, Extent $=83$ cents, Onset $=51$, vibrato jitter $=8,8$.

Los valores de normalidad para los parámetros acústicos del vibrato son: Rate: entre 5 y $7 \mathrm{~Hz}$, Extent: máximo 100 cents, Onset y Vibrato jitter no tienen rangos de normalidad establecidos, pero se considera deseable un valor bajo de onset y vibrato jitter.

\section{Plan de tratamiento}

El objetivo principal del entrenamiento vocal en el caso presentado es desarrollar un vibrato técnicamente adecuado (parámetros acústicos dentro de rangos normales). Este objetivo se llevo a cabo entrenando al paciente con un procedimiento denominado "Entrenamiento rítmico del vibrato" (desarrollado por el autor).

Esta práctica incluyó los siguientes aspectos:

1) Explicación del procedimiento y aspectos técnicos del vibrato

2) Disminución de la intensidad de la voz

3) Relajación de la musculatura fonatoria

4) Entrenamiento rítmico

5) Utilización de musculatura laríngea y abdominal

6) Naturalidad del vibrato

7) Integración en el repertorio

8) Reforzamiento visual 


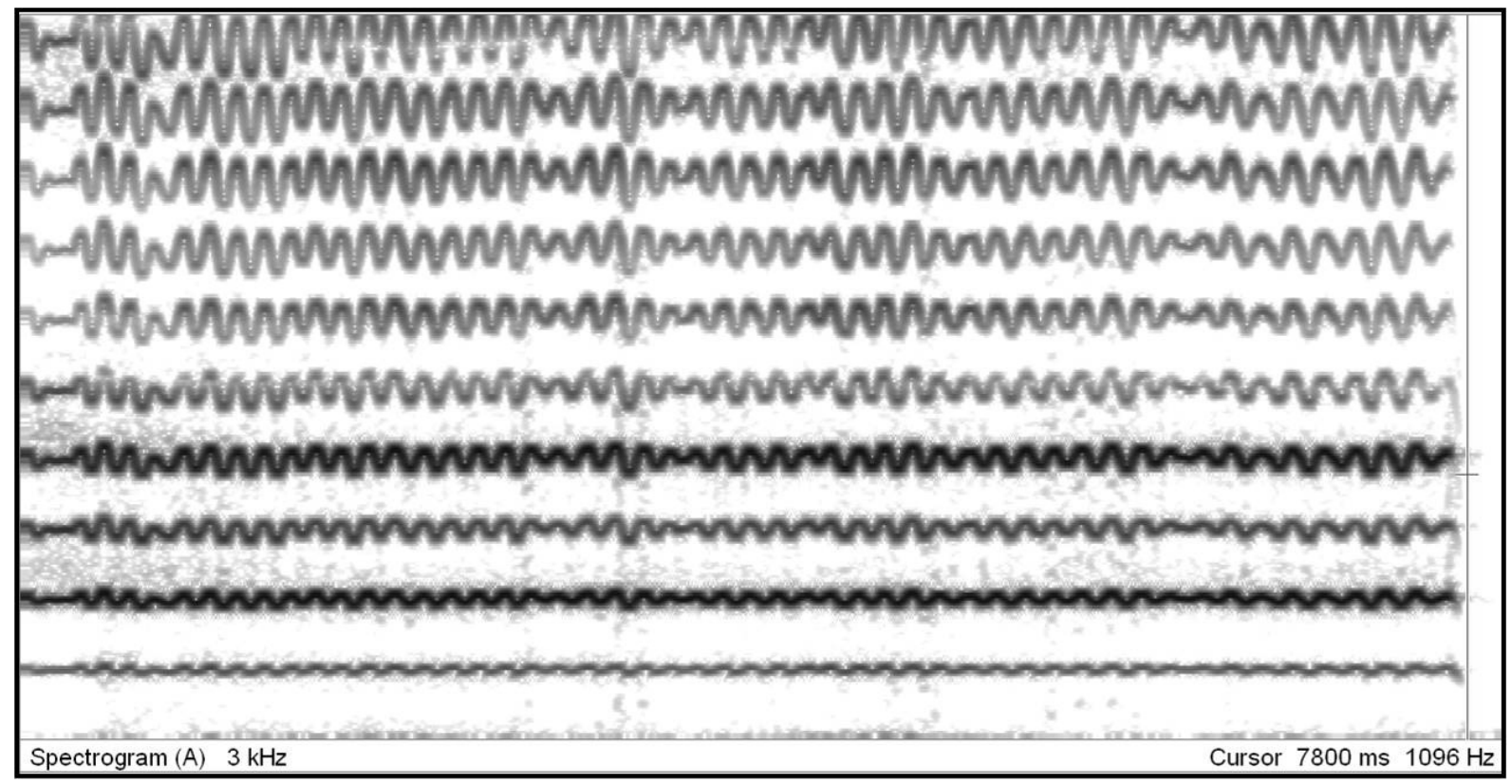

Figura 1 - "Espectrograma banda estrecha de la vocal /a/ cantada con un vibrato irregular.

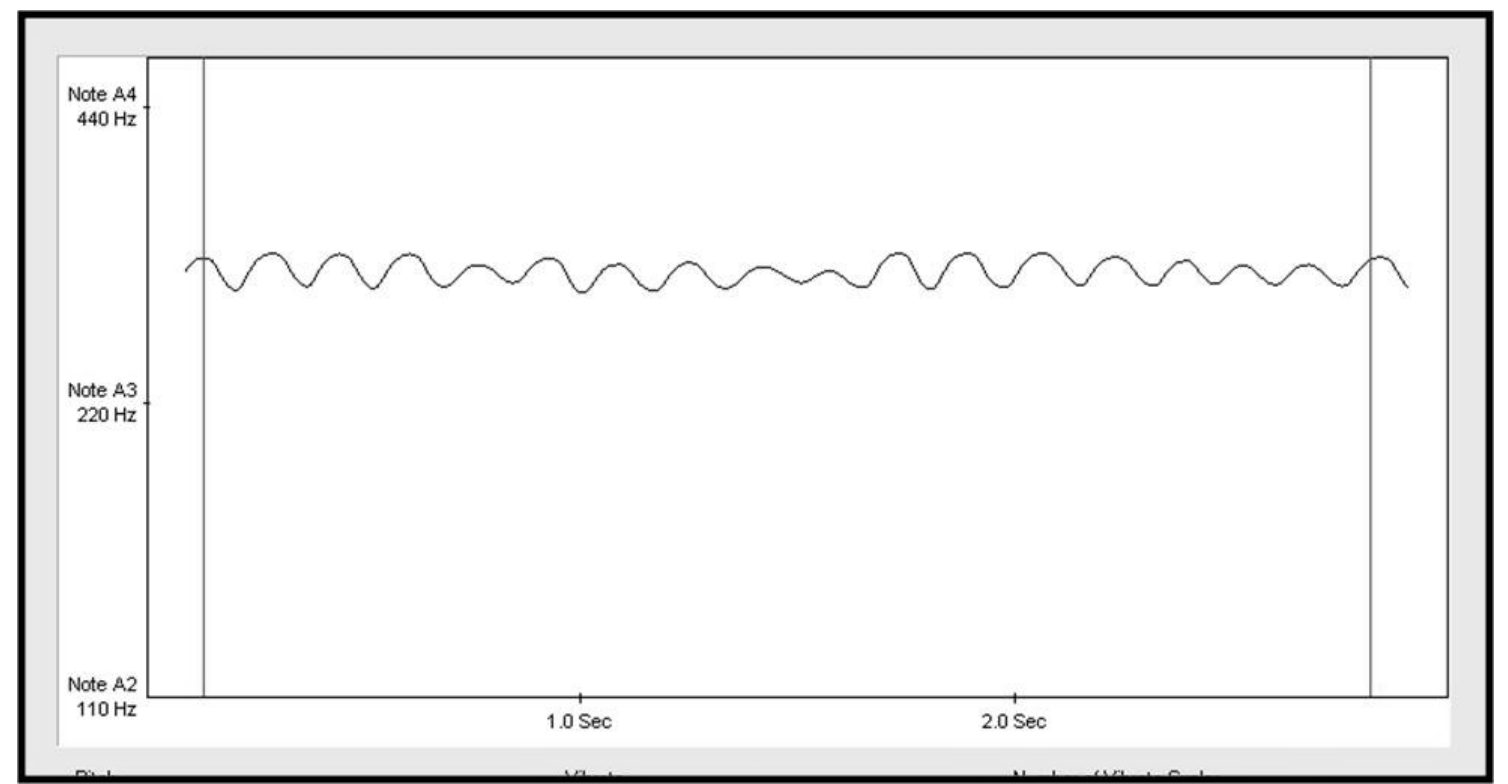

Figura 2 - "Vibrato extraído del cuarto armónico del espectrograma de la fig 1"

La base del "Entrenamiento rítmico del vibrato" es la subdivisión rítmica de un pulso o unidad de tiempo durante la emisión de una vocal con un tempo o velocidad constante.

Para lograr esto se comenzó con la explicación del procedimiento, la fisiología y los parámetros acústicos involucrados en la producción del vibrato.

Durante todo el entrenamiento se solicito mantener un volumen de voz medio y una emisión relajada utilizando la sensación de "garganta abierta".
Sin una emisión relajada y utilizando intensidad elevada se dificulta la realización del ejercicio puesto que éste considera como base la flexibilidad muscular y vocal.

Primero, se solicitó al sujeto mantener una emisión vocálica durante algunos segundos en un tono cómodo. En nuestro estudio se utilizó la vocal /a/ en $130 \mathrm{~Hz}$ (Do 2). El terapeuta paralelamente marcó un pulso constante utilizando un golpe con la mano o un metrónomo. El pulso debe tener una velocidad 
aproximada de negra $=60$, es decir, 60 pulsos por segundo. A continuación, el sujeto acentuó marcadamente con su voz cada pulso dado por el terapeuta en forma dinámica (aumento de intensidad) y tónica (aumento de tono). Dicho de otra forma, el sujeto marca el mismo pulso dado, con acentuaciones vocales durante la emisión de la vocal /a/ mantenida. Estas acentuaciones se acompañan de suaves contracciones abdominales.

El siguiente paso fue dividir el pulso rítmica y regularmente en dos subtiempos, es decir, por cada pulso que daba el terapeuta, el paciente hacia dos pequeñas acentuaciones con su voz. Una vez que el paciente logró realizar el ejercicio, se solicitó dividir el mismo pulso en 3 subtiempos y en forma posterior en 4. En otras palabras, se realizaron 3 y 4 acentuaciones vocales por pulso. Durante esta etapa se utilizó refuerzo visual a través del espectrograma de banda estrecha del software VoceVista. Esto facilitó el entendimiento y la realización del ejercicio.

El paso siguiente fue enfatizar el primer acento por sobre el resto. Cuando el paciente realizaba una división del pulso en cuatro acentos, solamente debía marcar el primero de ellos, teniendo los tres restantes menos énfasis de intensidad. Lo mismo se hizo para la subdivisión en dos y tres. La suave contracción abdominal debe ser levemente mayor para el primer acento en todos los casos. Mediante el refuerzo visual (Figura 3) se observa que la primera oscilación o acentuación tiene mayor extensión que el resto. Se ve además una línea divisoria en todos los armónicos cada dos, tres o cuatro acentuaciones según sea el caso ejercitado.

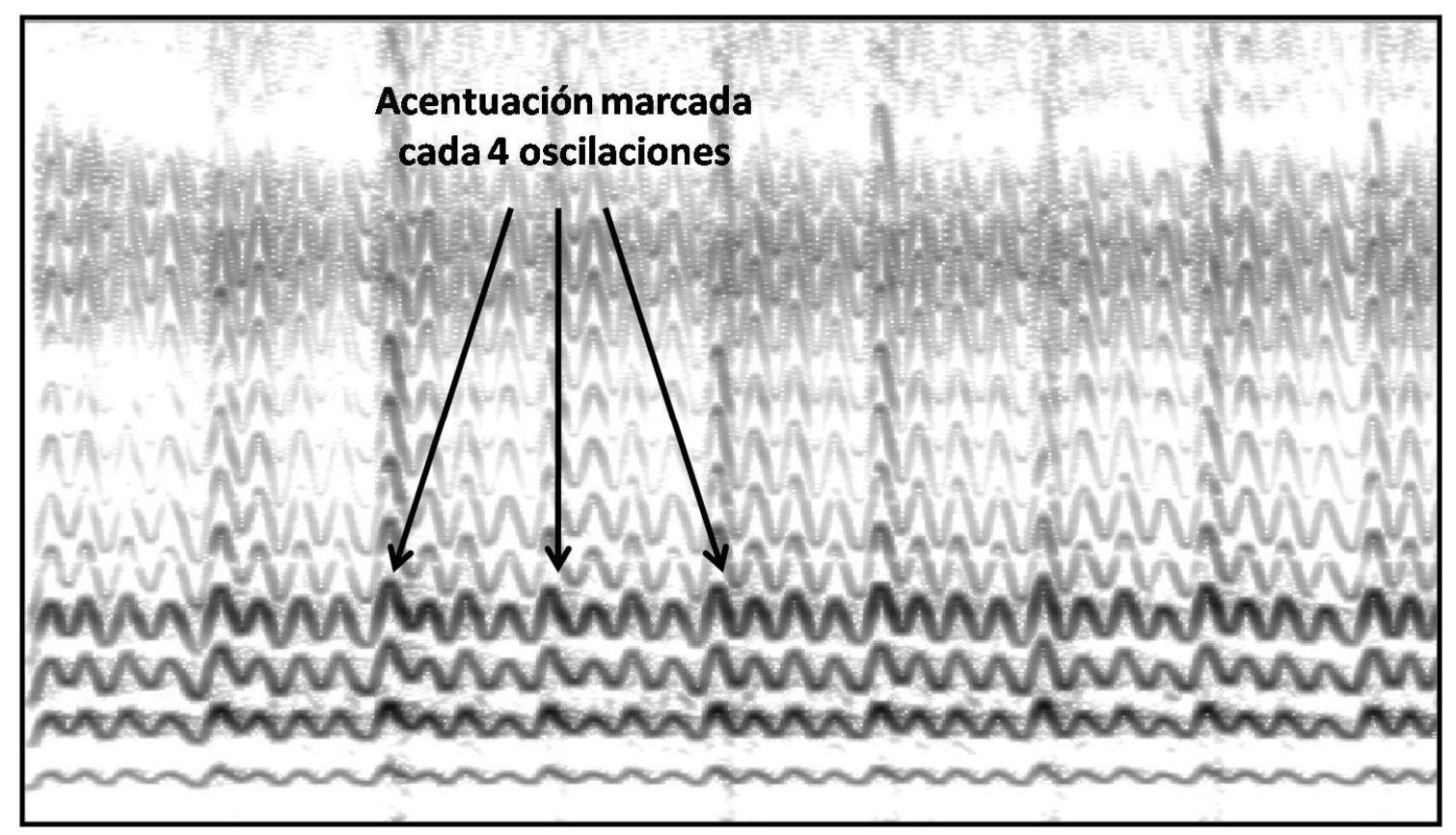

Figura 3 - "Ejercicio de vibrato realizado con acentuación marcada en la primera oscilación de 4 por cada pulso"

Una vez que el paciente manejó técnicamente el ejercicio, se realizaron cambios en el "tempo" o velocidad del pulso. Primero se disminuyó la velocidad del pulso y luego se aumentó. El rango de velocidad debe estar aproximadamente entre $80 \mathrm{y}$ 100 pulsos por segundo.

En la siguiente etapa se realizaron cambios en la vocal utilizada. Además de la vocal /a:/ se hizo uso de la /e:/, /i:/, /o:/, /u:/. En primera instancia el paciente debió hacer el ejercicio rítmico utilizando sólo una vocal y posteriormente se realizaron combinaciones de vocales, es decir, utilizar vocales diferentes consecutivas para cada pulso en una emisión. De esta forma, el sujeto debía mantener el pulso y las subdivisiones en forma regular teniendo la habilidad de hacer cambios de vocales durante el transcurso de la emisión (Figura 4).

A continuación se incluyeron cambios en tono (melodía). La emisión sostenida en un tono constante fue cambiada por una emisión mantenida utilizando intervalos musicales de segunda mayor, tercera mayor y quinta justa. A modo de 


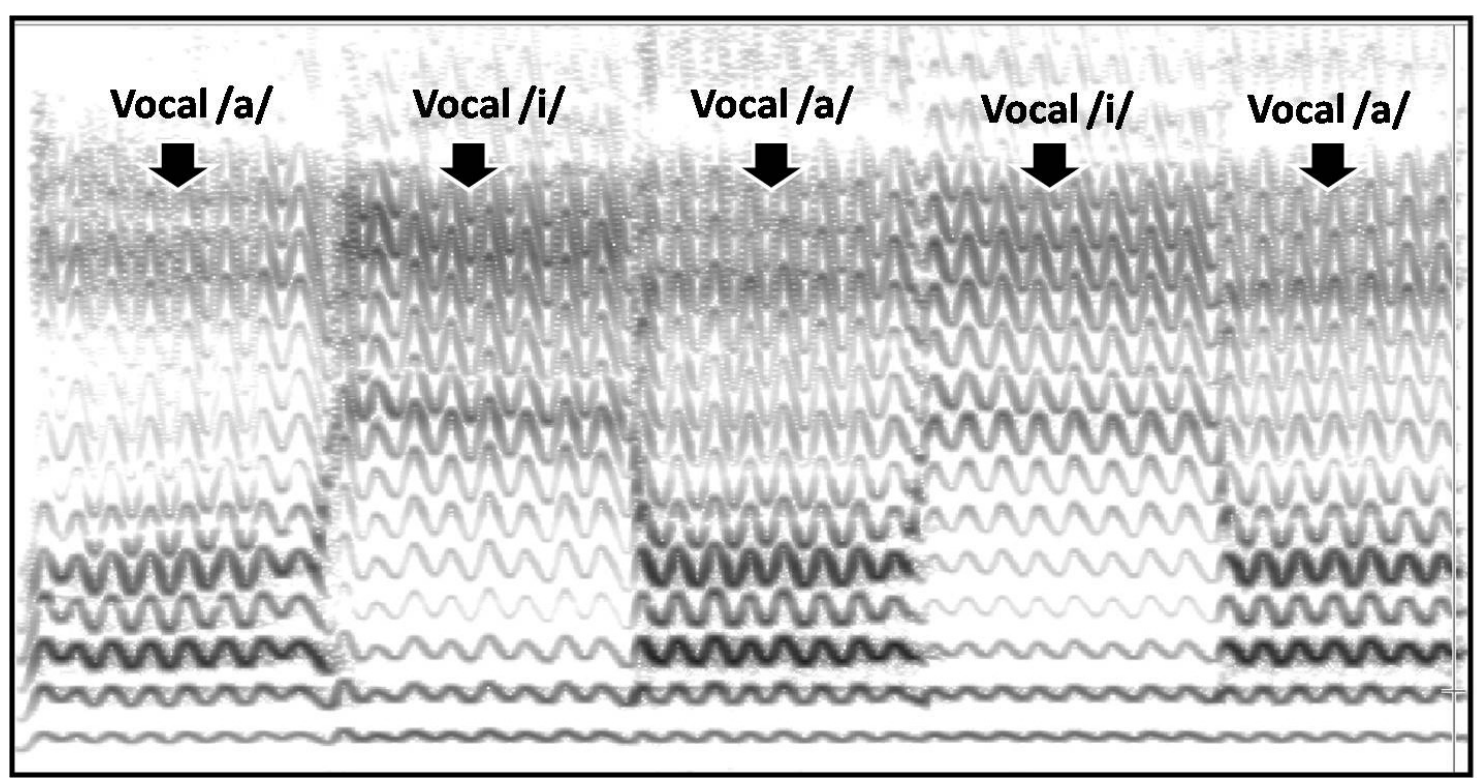

Figura 4 - "ejercicio de vibrato haciendo cambio de vocales. Secuencia de vocales /a/ e /i/"

ejemplo, si el paciente comenzaba la emisión en la nota Do, las dos siguientes notas fueron Re y Mi en forma ascendente (intervalos de segunda mayor) pudiendo hacerse inmediatamente la escala descendente $\mathrm{Mi}, \mathrm{Re}$, Do. De esta forma, se construyeron vocalizaciones simples a través de las cuales se debía mantener el ejercicio rítmico utilizando una o más vocales. La misma vocalización se realiza después en tonalidades más altas y más bajas (Figura 5).

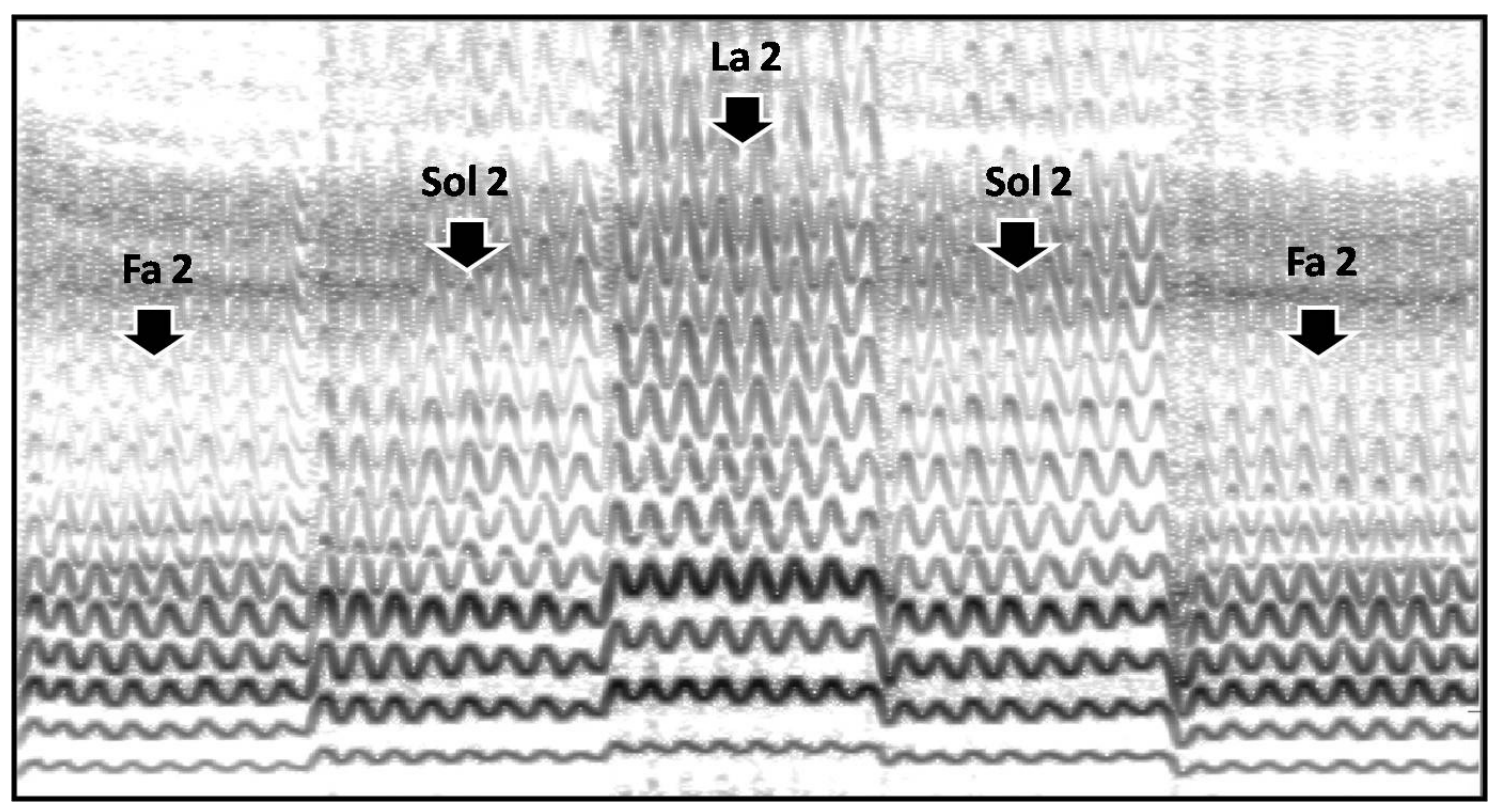

Figura 5 - "Espectrograma banda estrecha mostrando vocalización con ejercicio de vibrato" 
El paso siguiente fue entrenar la naturalidad del vibrato. Hasta el momento el paciente había realizado acentuaciones notorias siendo siempre la primera de cada pulso con mayor énfasis (acción de musculatura abdominal). Se solicito eliminar gradualmente el énfasis y hacer las acentuaciones cada vez menos notorias, de tal forma que las subdivisiones se asemejen a un vibrato regular, natural y técnicamente correcto. Muscularmente, se debe disminuir al mínimo la actividad abdominal y en forma posterior, eliminarla completamente. En todo momento se le solicitó al sujeto mantener una intensidad media y utilizar la sensación de "garganta abierta". En el espectrograma de banda estrecha (refuerzo visual) se ve que al disminuir las acentuaciones, las oscilaciones de los armónicos se asemejan cada vez una a la otra, apareciendo éstas como vibrato natural (Figura 6).

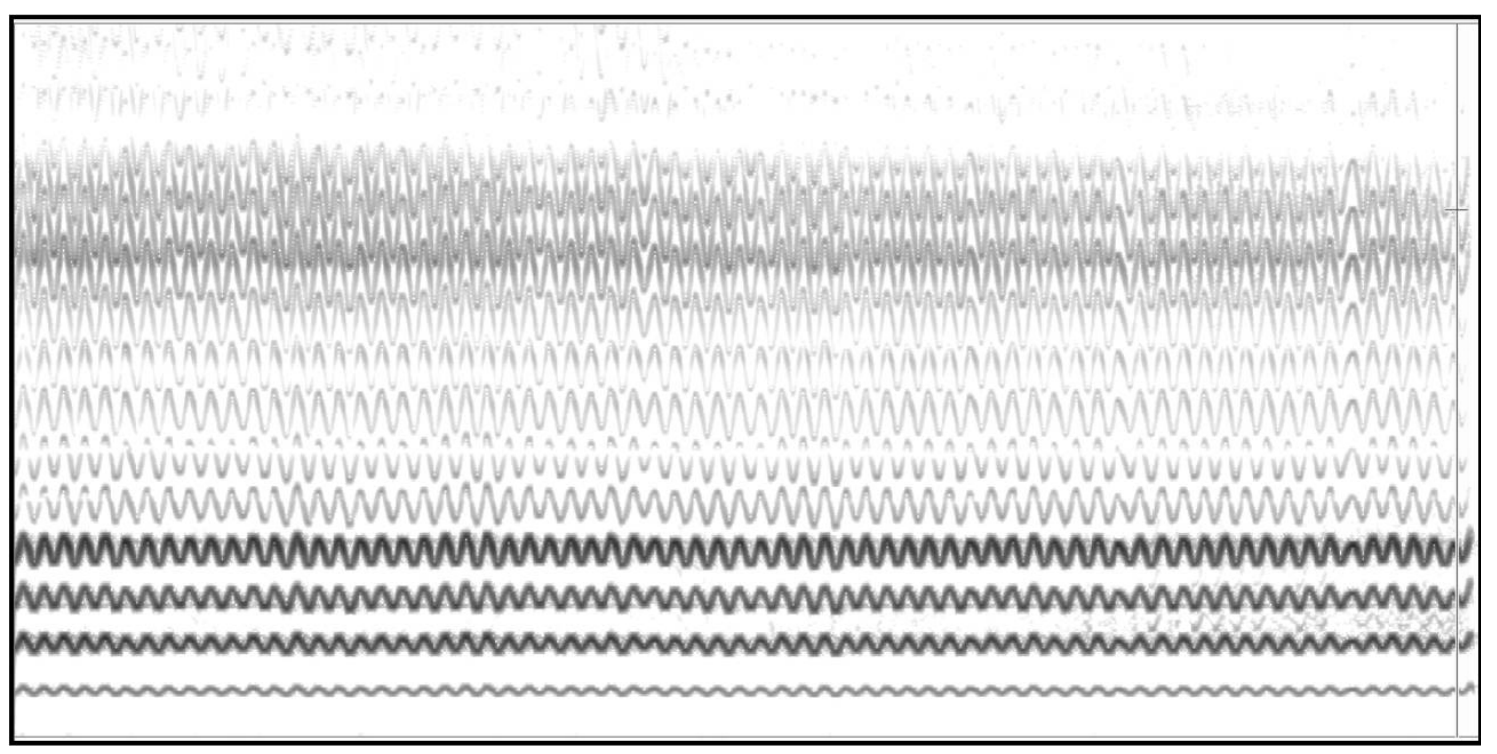

Figura 6 - "Espectrograma banda estrecha del vibrato del paciente después del entrenamiento"

La última etapa del entrenamiento fue la integración de la emisión con vibrato en el repertorio musical. Se escogieron dos arias que él había cantado durante los primeros años de estudio: "Ombra mai fu" y "Amarilli". La utilización de estas arias se debe a dos razones, ambas presentan notas de larga duración donde se puede poner en práctica el ejercicio enseñado, y no requieren ser cantadas con gran intensidad.

El entrenamiento del vibrato se realizó durante un mes y medio, con una frecuencia de 1 sesión a la semana (total 6 sesiones). Cada sesión tuvo una duración de 35 minutos. Durante el tiempo transcurrido entre dos sesiones el paciente realizó los ejercicios en su hogar dos veces al día durante 15 minutos cada vez.

La evolución del entrenamiento del vibrato fue evaluada objetivamente en la primera y la última sesión. Dicho procedimiento se realizo grabando la voz del sujeto en una sala con escaso ruido ambiental. La señal de voz fue capturada directamente en un computador portátil utilizando un preamplificador modelo M-Audio pro USB y un micrófono Shure PG48. El micrófono fue situado a $10 \mathrm{~cm}$, de los labios del paciente, el cual se encontraba en posición sentado. Se analizaron dos emisiones: una vocal /a/ sostenida en un tono cómodo para el paciente e intensidad media, y la primera vocal /a/ de la palabra "Amarilli" de la canción del mismo nombre (Compositor: Giulio Caccini). Se escogió esta última vocal ya que su emisión tiene una duración de tres tiempos, suficiente para realizar el análisis acústico con el software VoceVista.

Los resultados del entrenamiento del caso presentado serán los valores objetivos de los rasgos acústicos del vibrato previamente señalados (rate, extent, onset y vibrato jitter).

Este articulo fue aprobado por la comisión de ética de investigación de la Escuela de Fonoaudiología de la Universidad de Chile con el numero 20/2009. 


\section{RESULTADOS}

Después de seis sesiones de trabajo, más la práctica diaria en casa se logró que el paciente pudiera producir consciente y voluntariamente un vibrato regular (menor valor de vibrato jitter), y un extent dentro de rangos normales. Los valores de los parámetros acústicos del vibrato medidos en la última sesión de entrenamiento fueron: Emisión vocal /a/ sostenida: Rate $=6 \mathrm{~Hz}$, Extent $=85$ cents, onset $=49 \mathrm{~ms}$. y vibrato jitter $=4,2 \%$. Emision vocal de la palabra "amarilli": Rate $=6 \mathrm{~Hz}$, Extent $=82$ cents, onset $=50 \mathrm{~ms}$. y vibrato jitter $=4,4 \%$.

Considerar que los valores de normalidad para los parámetros acústicos del vibrato son: Rate: entre 5 y $7 \mathrm{~Hz}$, Extent: máximo 100 cents, Onset y Vibrato jitter no tienen rangos de normalidad establecidos. En la Figura 7 se observa parte del aria "Amarilli" donde la nota más larga corresponde a la vocal /a:/.

\section{DISCUSIÓN}

Este trabajo presenta el caso de un estudiante de canto lirico con problemas en vibrato. Considerando que el vibrato es uno de los aspectos más importantes en un cantante entrenado, es de gran importancia que los parámetros acústicos de éste se encuentren dentro de los rangos estéticos y técnicos de normalidad ${ }^{8}$. En el caso presentado, este objetivo se concretó a través del procedimiento denominado "Entrenamiento rítmico del vibrato".

Existen dos claras posturas en relación al desarrollo del vibrato. Una de ellas plantea que es inapropiado intentar cambiar los rasgos del vibrato en forma voluntaria. Los adeptos a este planteamiento señalan que el vibrato debe ser cultivado, no producido. En otras palabras, se propone que el vibrato se debe expresar por si sólo producto de una técnica vocal globalmente correcta ${ }^{3}$.

Por otra parte, otros profesores si creen posible y adecuado desarrollar o corregir un vibrato inapropiado en sus alumnos, a través de ejercicios específicos y dirigidos. Existen datos experimentales que muestran la posibilidad de los cantantes de poder cambiar el valor del rate ${ }^{24}$. Seidner ${ }^{25}$ señala que muchos cantantes deben ajustar los parámetros del vibrato dependiendo de factores técnicos e interpretativos. Es así, por ejemplo, que un cantante de ópera debe reducir o eliminar el extent si desea cantar música antigua.

En el caso presentado, el alumno de canto cursaba su 5 año de educación formal. Aun cuando su técnica vocal global era correcta y acorde a su nivel de estudios, el vibrato no siguió un desarrollo adecuado. Considerando esto, cabe plantear algunas preguntas: ¿Debemos esperar indefinidamente que el vibrato se adquiera en forma natural? o ¿Podemos en algunos casos de vibrato incorrecto, crearlo y/o corregirlo en forma voluntaria y específica?

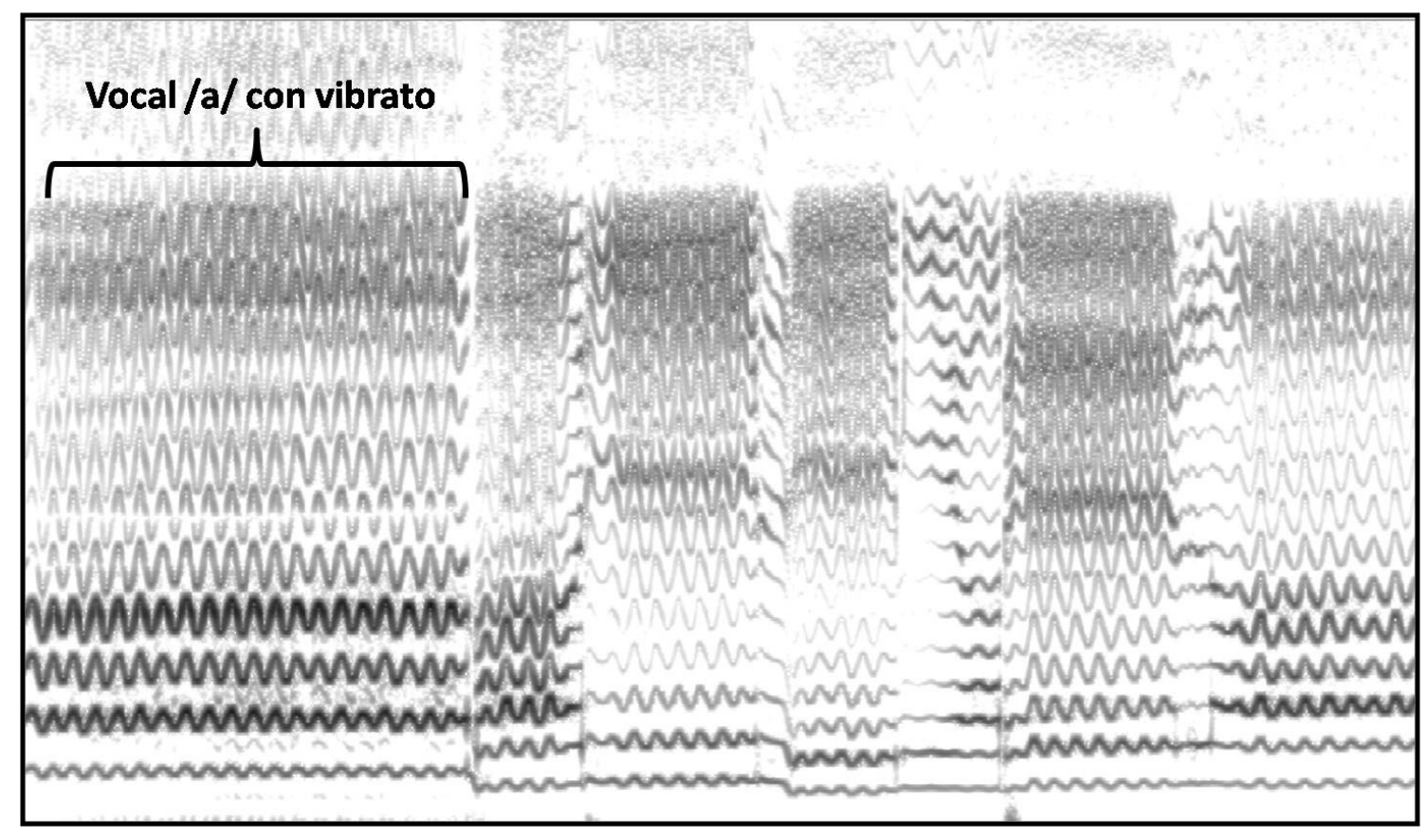

Figura 7 - "Espectrograma banda estrecha del comienzo del aria amarilli" 
El paciente reportado necesitaba rápida ayuda en relación a la corrección de su vibrato ya que este aspecto le impedía tener una voz comercialmente competitiva de acuerdo a los estándares del canto clásico, a pesar de su gran desarrollo técnico vocal general.

Si bien lo ideal es esperar un desarrollo natural del vibrato, en este trabajo se plantea que en algunos casos ese desarrollo no se produce a pesar de la buena técnica vocal y por lo tanto si se debe intervenir en forma específica.

Kirkpatrick ${ }^{23}$ describe tres alteraciones particulares del vibrato con sus respectivas soluciones a través de ejercicios específicos. El autor propone ejercicios dirigidos principalmente al control de la presión subglótica y el flujo de aire entre las cuerdas vocales, señalando que el balance de estos dos factores afecta la producción del vibrato.

Por otra parte, GuLi-de ${ }^{26}$ describe soluciones prácticas para cantantes que no pueden lograr completamente las características del vibrato según el estilo musical. Señala que las principales características de un vibrato anormal son la irregularidad y el rate disminuido o aumentado.

Un aspecto importante abordado en el "Entrenamiento rítmico del vibrato" es la utilización tanto de la musculatura laríngea, como la abdominal. Si bien, el vibrato es generalmente atribuido a la actividad del músculo CT ${ }^{15,17}$, existen evidencias de que la musculatura abdominal y diafragmática pueden tener una participación importante en la producción de vibrato ${ }^{18}$. Como ya se señaló, el vibrato puede ser separado en dos tipos de producción. La primera se relaciona con la producción rítmica de pulsaciones de presión subglótica producida por contracciones de los músculos abdominales y la otra, que es la mediada por la laringe, en la cual la modulación de FO resulta principalmente de la modulación de la actividad del CT.

En el presente planteamiento, se consideran dos elementos importantes: la acentuación tónica y la dinámica. La primera es el énfasis dado a la emisión mediante un pequeño cambio en el tono y probablemente atribuida mayormente a una actividad laríngea del CT. Por otra parte, la acentuación dinámica es el énfasis determinado por un aumento de la intensidad del sonido, dado en este caso por el leve aumento de la presión subglótica determinada por las pequeñas contracciones de la musculatura abdominal.

Tal como afirma Laukkanen ${ }^{21}$, en el presente trabajo se plantea que los cantantes producen vibrato utilizando uno de los dos tipos de producción o la combinación de ambas en distinto grado. En el "Entrenamiento rítmico del vibrato" se hace uso de las dos, cuidando que la actividad abdominal sea mínima y que en ningún caso tenga predominancia por sobre la actividad laríngea. Shipp ${ }^{20}$ señala que le método abdominal de producción de vibrato es el más útil para controlar el rate, justamente uno de los parámetros fuera de normalidad en el paciente reportado.

Por otra parte, un aspecto importante en el proceso, es la disminución gradual de la contracción abdominal hasta llegar a cero. Si no se elimina esta actividad, el cantante probablemente dependerá de ella para la producción del vibrato. Esto podría además a producir un vibrato con poca naturalidad.

Durante la realización de los ejercicios se dio especial importancia a la relajación de la musculatura fonatoria. Gu Li-de ${ }^{26}$ señala que la hipertensión de cualquier músculo laríngeo puede afectar la calidad de la voz significativamente.

Uno de los aspectos considerados en el entrenamiento de la relajación de la musculatura fonatoria fue la técnica de la "garganta abierta". Esta técnica es un concepto que históricamente se ha planteado como una forma de describir libertad, perdida de tensión y mejor cualidad vocal. De acuerdo con la literatura la "garganta abierta" es una maniobra compleja que involucra una instrucción pedagógica que trae como resultado un sonido especifico. Existen estudios que asocian esta técnica con el vibrato. Es así como Mitchell ${ }^{12}$ encontró que el extent y onset se ven afectados por el grado de utilización de la "garganta abierta". Al no hacer uso de esta técnica ambos parámetros fueron mayores y fuera de los rangos aceptados como adecuados.

La disminución de la intensidad también se realizó como una forma de mantener una fonación libre de tensiones. Recordar que el sujeto se caracterizó por utilizar la voz en la mayoría de los casos con una intensidad aumentada y posiblemente la innecesaria hiperaducción cordal y tensión laríngea que esto conlleva. Al disminuir la excesiva intensidad de la voz se permitió mayor flexibilidad en la realización de los ejercicios.

Después del entrenamiento, el vibrato producido por el sujeto fue técnicamente correcto en rate, extent y regularidad, pero este no se percibía absolutamente natural debido a que el alumno aún debía pensar conscientemente en las subdivisiones. En consideración de esto, la naturalidad del vibrato desarrollado con esta técnica, podría uno de los aspectos cuestionables, sin embargo hemos tenido éxito en tres casos anteriores al reporte planteado en este trabajo en donde el vibrato ha llegado a tener una naturalidad adecuada. Además, en una evaluación realizada un mes después del término del entrenamiento, el vibrato del paciente fue considerablemente más natural desde el punto de vista perceptual. 
Finalmente, cabe destacar que el entrenamiento del vibrato, además de abordar los aspectos técnicos, debiera considerar en una etapa más avanzada y posterior los factores estilísticos dependiendo del estilo musical, tipo de música y periodo en que la música que se está interpretando fue compuesta $^{27-29}$. Dependiendo de estos y otros elementos podremos encontrar diferentes tipos de vibrato $^{28}$. Otro elemento que determinara la presencia y tipo de vibrato es el hecho de cantar en un ensamble, coro o como solista. Por otra parte, las exigencias del director también constituirán un factor en la regulación del vibrato ${ }^{30}$.

\section{CONCLUSIÓN}

El vibrato ha sido reconocido ampliamente como uno de los rasgos esenciales de una buena calidad vocal en el canto clásico. Existen posturas que plantean que el vibrato nunca de debe ser entrenado o creado voluntariamente y otras que consideran adecuada la corrección y entrenamiento en forma específica. En el presente trabajo se propuso un procedimiento denominado "Entrenamiento rítmico del vibrato" el cual se basa principalmente en la subdivisión rítmica de un pulso o unidad de tiempo durante la emisión de una vocal con un tempo o velocidad constante. El paciente tratado logro después de 6 sesiones de entrenamiento, controlar y corregir los problemas de regularidad y rate presentados. Después de terminar el entrenamiento, el sujeto continuó mejorando la calidad de su vibrato, principalmente la naturalidad de éste. En este trabajo se plantea que si posible el entrenamiento y/o corrección del vibrato en forma dirigida a través de ejercicios específicos destinados a ese objetivo. El apoyo visual cumple un rol importante en el resultado de este entrenamiento, a través de este, el alumno toma conciencia y modifica los parámetros alterados del vibrato en corto tiempo.

\begin{abstract}
Background: vocal vibrato is one of the most important acoustic, technical and stylistic features to characterize the voice quality in classical singers. Technically, vibrato is a pulsation of pitch, usually accompanied with synchronous pulsations with intensity and timbre. Vibrato can be described acoustically by the rate, extent and periodicity of frequency modulations and amplitude modulations of the voice. Procedures: an opera singer student, 25 -year-old, lyric baritone, with normal voice from the perceptual point of view. The correction of vibrato is carried through by using the method "Rhythmic vibrato training". One produces a rhythmic pulse subdivision or unit of time during a vowel emission with "time" or speed constant. Subsequently, there is a variation in pulse speed, vowels and melody. The acoustic parameters of vibrato are evaluated objectively before and after training. Results: the patient succeeded to produce a vibrato with rate, extent, and regularity within the normal ranges. Vibrato production was carried through in a conscious directed way by using abdominal and laryngeal activity at the same time. The vibrato continued improving after the training had been completed. Conclusions: it is possible to train and correct the vibrato in a targeted way through specific exercises, intended for such purpose. The visual biofeedback plays an important role on the outcome.
\end{abstract}

KEYWORDS: Voice; Voice Quality; Voice Training

\section{REFERÊNCIAS}

1. Seashore C. The vibrato. University of lowa studies in the psychology of music. vol 1. lowa City: University Press; 1932.

2. Hirano M, Hibi S, Hagino S. Physiological aspects of vibrato. In: Dejonckere P, Hirano M, Sundberg J. Vibrato. San Diego, CA: Singular Publishing Company; 1995. p. 9-33.

3. Murbe D, Zahnert T, Kuhlisch E, Sundberg J. Effects of professional singing education on vocal vibrato, a longitudinal study. J Voice. 2007; 21:683-8. 4. Sundberg J. The science of the singing voice. DeKalb: Northern Illinois University Press; 1987.

5. Sundberg J. Acoustic and psychoacoustic aspects of vocal vibrato. In: Dejonckere P, Hirano M, Sundberg J. Vibrato. San Diego, CA: Singular Publishing Company; 1995. p. 35-62.

6. Howes P, Callaghan J, Davis P, Kenny D, Thorpe $W$. The relationship between vibrato characteristics and perception in Western operatic singing. $J$ Voice. 2004; 18:216-30. 
7. Robison C, Bounous B, Bailey R. Vocal beauty: a study proposing its acoustical definition and relevant causes in classical baritones and female belt singers. NATS J. 1994; 51:19-30.

8. Dromey C, Carter N, Hopkin A. Vibrato rate adjustment. J Voice. 2003; 17:168-78.

9. Diaz J, Rothman H. Acoustical comparison between samples of good and poor vibrato in singers. J Voice. 2003; 17:179-84.

10. Cecconello L, Guzmán M. Correlación entre valoración perceptual y parámetros acústicos del vibrato en tenores famosos. XIX ENT World Congress-IFOS; 2009 Brazil. 2009 Junio 1-5; São Paulo, Brazil. [CD Room].

11. Ekholm E, Papagiannis G, Chagnon F. Relating objective measurements to expert evaluation of voice quality in Western classical singing: critical perceptual parameters. J Voice. 1998; 12:182-96.

12. Mitchell H, Kenny D. The impact of "open throat" technique on vibrato rate, extent and onset in classical singing. Logoped Phoniatr Vocol. 2004; 29:171-82.

13. Horii $Y$, Hata K. A note on phase relationships between frequency and amplitude modulations in vocal vibrato. Folia Phoniatr. 1988; 40:303-11.

14. Schutte $H$, Miller D. Acoustic details of vibrato cycle in tenor high notes. J Voice. 1991; 5:217-23.

15. Imaizumi $S$, Saida $H$, Shimura $Y$, Hirose $H$. Harmonic analysis of the singing voice. Stockholm: Royal Swedish Academy of Music; 1993.

16. Dromey C. Laryngeal-level amplitude modulation in vibrato. J Voice. 2009; 23:156-63.

17. Horii Y. Acoustic analysis of vocal vibrato: a theoretical interpretation of data. J Voice. 1989; 3:36-43.

18. Hirano M, Vennard W, Ohala J. Regulation of register, pitch and intensity of voice. An electromyographic investigation of intrinsic laryngeal muscles. Folia Phoniatr. 1970; 22:1-20.

19. Appelman D, Smith E. Cinefluorographic and electromyographic observations of abdominal muscular function in its support of vibrato.
Transcripts of the fourteenth symposium: care of the professional voice; 1985 set 79-82; New York: The Voice Foundation. p. 79-82

20. Shipp T, Doherty E, Haglund S. Physiologic factors in vocal vibrato production. J Voice. 1990; 4:300-4.

21. Laukkanen A, Vilkman E, Unto K. Aspects of the physiological sources of vocal vibrato: a study of fundamental period-synchronous changes in electroglottographic signals obtained from one singer and two excised humanlarynges. Scan J Log Phon. 1992; 17:87-93.

22. Shipp T, Leanderson R, Sundberg J. Some acoustic characteristics of vocal vibrato. J Res Sing. 1980; 4:18-25.

23. Kirkpatrick A. Teaching methods for correcting problematic vibratos: using sustained dynamic exercises to discover and foster healthy vibrato. $\mathrm{J}$ Sing. 2008; 64:551-6.

24. King J, Horii Y. Vocal matching of frequency modulation in synthesized vowels. J Voice. 1993; 7:151-7.

25. Seidner W. Dependence of vibrato on pitch, musical intensity, and vowel in different voice classes. In: Dejonckere P, Hirano M, Sundberg J. Vibrato. San Diego, CA: Singular Publishing Company; 1995. p 63-82.

26. Gu Li-de. Inducing factors and treatment for vibrato problems Vibrato. In: Dejonckere P, Hirano M, Sundberg J. Vibrato. San Diego, CA: Singular Publishing Company; 1995. p 145-9.

27. Sublett V. Vibrato or nonvibrato in solo and Choral singing: is there room for both? J Sing. 2009; 65:539-44.

28. Isherwood N. Vocal vibrato: new directions. J Sing. 2009; 65:271-83.

29. Jorold B. Distinguishing between artificial and natural vibrato in premodern music. J Sing. 2006; 63:161-7.

30. Olson M. Vibrato v/s Nonvibrato: the solo singer in the collegiate choral ensemble. J Sing. 2008; 64:561-4.

doi: $10.1590 / S 1516-18462010005000072$

RECEBIDO EM: 31/08/2009

ACEITO EM: 30/03/2010

Endereço para correspondência:

Marco Guzmán

Escuela de Fonoaudiología, Facultad de Medicina

de la Universidad de Chile

Av. Independencia 1027, Independencia,

Santiago, Chile

E-mail: guzmanvoz@gmail.com 